\title{
Shallow-water spinal injuries - devastating but preventable
}

\author{
A J Vlok, I Petersen, R N Dunn, J Stander
}

Background. Shallow-water diving injuries have devastating consequences for patients and their families, requiring intensive use of resources in both the acute and rehabilitative phases of injury. With the final clinical outcome often poor, the question is raised as to whether a target group can be identified for whom to implement a preventive programme.

Objective. To evaluate the demographics, clinical features and outcomes of shallow-water diving injuries in an acute spinal cord injury (ASCI) unit.

Materials and methods. All patients admitted to the ASCI unit with diving-related injuries were entered into the study. Data regarding demographics, injury profile and subsequent management were collated. All case notes and X-rays were reviewed. Ethical approval was obtained.

Results. Forty-six patients were reviewed from 19 April 2003 to 8 February 2009. A steady annual increase in diving injuries was noted. A very specific patient profile was identified: $91 \%$ male incidence, average age 23 years, $37 \%$ admitted alcohol use, with a summer-time prevalence. Compression-flexion type injuries were most prevalent, with an orthopaedic level of $\mathrm{C} 5$ and neurological level of $\mathrm{C} 4$ being the most common injury sites. A third of diving injuries occurred in the sea, $20 \%$ in swimming pools, $20 \%$ in rivers, $11 \%$ in tidal pools and $4 \%$ in dams.

Conclusion. A very specific patient profile was identified, and the severity of shallow-water diving injuries was confirmed. No current preventive programme exists except for a single television advertisement. These data will be used to motivate further educational and preventive programmes for reducing the incidence of diving-related injuries.

S Afr Med J 2010; 100: 682-684.
Shallow-water diving injuries are endemic in South Africa. They have catastrophic neurological sequelae in the younger population, at tremendous personal cost and a burden to society, medical service providers and social support structures. The management requires intensive resources in the acute and rehabilitation phases. Divingrelated cervical injuries, often leading to quadriplegia and death, constitute a subgroup of cervical spine injuries. We studied divingrelated cervical spine injuries with the aim of identifying a specific South African target group for whom to implement a prevention programme.

\section{Materials and methods}

The Acute Spinal Cord Injury (ASCI) Unit at Groote Schuur Hospital (GSH), which provides a regional provincial service and also deals with referrals from outside the Western Cape province, was established in March 2003 after having been moved from Conradie Hospital owing to provincial restructuring. It comprises 6 ventilated beds and 15 ward beds which are supplemented by physiotherapists, occupational therapists and social workers. The ASCI Unit provides acute physiological and orthopaedic stabilisation and is a closed referral unit. Polytrauma patients, or patients with unresolved head injuries, are excluded. Personal, demographic and surgical data from all patients are entered into a database. GSH provides the acute

Acute Spinal Cord Injury Unit, Groote Schuur Hospital and University of Cape Town

A J Vlok, MB ChB, MMed (Neurosurg), FCNeurosurg (SA)

R N Dunn, MB ChB, MMed (Orth), FCOrth (SA)

J Stander, $\mathrm{MB} \mathrm{ChB}$

Department of Orthopaedic Surgery, Tygerberg Hospital and Stellenbosch University, $W$ Cape

I Petersen, MB ChB service, and rehabilitation is at the Western Cape Rehabilitation Centre (WCRC) in Mitchells Plain.

We identified all patients with 'diving' in the aetiology from a database and reviewed their case notes and radiographs. Details collected included age, gender, injury date and place, diving technique and alcohol consumption. The American Spinal Injury Association (ASIA) score ${ }^{1}$ was used to assess the neurological status of each patient at admission and discharge. Spinal cord injuries were classified either as complete or incomplete based on the status of the lowest sacral segment, with 'complete' referring to no motor or sensory function in that segment, and 'incomplete' as sacral sparing being present. The neurological level was defined as the most caudal segment with $5 / 5$ power and normal sensation in both modalities of pinprick and light touch.

Injury data included how the patient was removed from the water, delay experienced by the spinal injury unit, orthopaedic and neurological level, ASIA score, surgical data and procedure, and discharge destination. Clinical follow-up data were reviewed and complications were noted. The data were also compared with international literature. Ethical approval was obtained prior to data collection. All patients who were referred to the WCRC were also followed up.

\section{Results}

Forty-six patients were identified during the period 19 April 2003 - 8 February 2009. A steady rise in patient numbers was noted, with a peak of 14 patients in 2008 (Fig. 1). School and university holiday periods included $57 \%$ of the injuries; there was a definite summertime prevalence; $91 \%$ of the patients were male, with an average age of 23 years at the time of injury; and $37 \%$ of all the injured patients admitted to alcohol consumption. Twenty patients were injured in the sea, 13 in swimming pools, 7 in rivers, and 4 in tidal pools; in 2 cases, the location was not documented. The average time for transfer to the ASCI Unit was 3.6 days, with a mean time of 2 days.

The ASIA scoring system, using modalities of light touch, pinprick sensation and motor strength, revealed a slight improvement in all modalities (motor strength from 33 to 43 , light touch from 57 to 68 , 


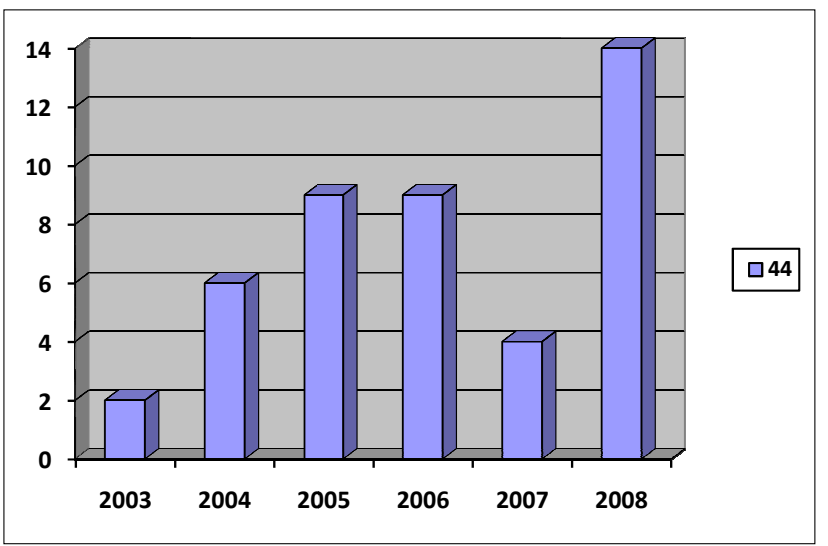

Fig. 1. Annual incidence of diving-related cervical spine injuries.

pinprick sensation from 54 to 65 ); 25 patients had an incomplete neurological status, 13 were neurologically complete and 8 had no neurological fallout. The most common orthopaedic level was C5, and $\mathrm{C} 4$ was the most common neurological level of injury.

Compression-flexion type injuries were the most common $(20$ patients) followed by burst fractures (17 patients) and bi- and unifacet dislocations in 4 . All bifacet dislocations occurred at level C4, with $50 \%$ C4 and 50\% C5 in unifacet dislocations. Most compression flexion and burst fractures occurred at C5 level.

The financial implications of these injuries were calculated on the basis of length of stay and surgical procedures performed. The total stay time in the unit was 1381 days, averaging to 30 days per patient. This equates to R150 108 per patient excluding the cost of medicine, surgery and rehabilitation. A total of 27 patients underwent surgery (22 anterior, 6 posterior and 1 anterior and posterior procedure). Total surgical cost was R810 000, making the total financial burden for diving injuries in this study R7 715000 , excluding medicine and rehabilitation costs. Thirty per cent of patients were discharged home, with $61 \%$ transferred to a specialised rehabilitation unit.

\section{Discussion}

The severity of shallow-water cervical spine injuries has been reported in studies from around the globe. They all identify a very specific group - a young male in summer who is probably inebriated..$^{2-5}$ Our study confirmed these specific patient characteristics, with a $91 \%$ male domination, an average age of 23 years, clear summer-time prevalence and a $37 \%$ admission of alcohol consumption. The young male diving injury victim is a common finding in studies from Italy ( $97 \%$ male, average age 22 years) ${ }^{4}$ and the USA ( $87 \%$ male, average age 24 years). ${ }^{2}$ The summer-time prevalence is confirmed in the USA (82\% injuries from June to August) $)^{2}$ and Greece (100\% injuries from May to September). ${ }^{3}$ The $37 \%$ admitted alcohol consumption in this study correlates well with other international studies $\left(49 \%^{2}\right.$ and $47 \%,{ }^{5}$ respectively). Such a distinct patient population paves the way for an effective prevention programme. No such programme currently exists, with the exception of a television advertisement initiated by our unit. The steady increase in cervical spine injuries owing to diving incidents since 2003 (Fig. 1) confirms the need in South Africa for such a programme.

Swimming pools and the sea are the most common locations of injury. Only 2 patients in this study were removed from the water by a lifeguard, and $22 \%$ of patients reported that they were injured during their first visit to the location of injury. A study of 341 patients in the USA established that, since $1973,44 \%$ of people were injured during their first visit to the location of injury and that weekends accounted for $51 \%$ of their injuries. ${ }^{2}$ A diving-related study from Canada showed 203 patients interviewed with only $37 \%$ aware that they could have sustained a cervical injury from diving. ${ }^{5}$ It is surprising that even a developed country does not have a prevention programme effective enough to increase the awareness of cervical injuries caused by diving.

Another common factor from around the world is the severity of the injuries. In an Italian study of 65 patients, 54\% were neurologically complete. ${ }^{4}$ In our study, 13 out of $46(28 \%)$ were neurologically complete, and 25 out of 46 (54\%) had an incomplete neurological status. The neurological status was assessed by means of the ASIA scoring system using the modalities of light touch, pinprick touch and motor strength. The score was assessed on admission and discharge and compared among the different modalities (Fig. 2). All 3 modalities increased by around 10 points, reflecting only radicular recovery, usually at the level of cord injury after spinal shock has reversed. This poor recovery indicates the severity of the injuries sustained in this study.

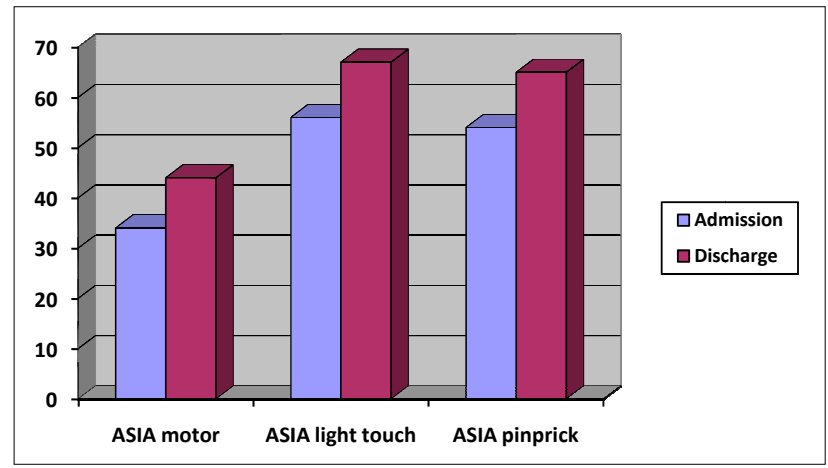

Fig. 2. Neurological (ASIA) scores of patients on admission and discharge.

Compression flexion-type injuries were the most common type of bony injury sustained, which would be typical for someone diving onto a firm surface and striking it with their forehead. Burst fractures were also commonly found with this mechanism of injury. Unifacet and bifacet dislocations, both known for potential severe neurological fallout, were 4 each. The most common orthopaedic level of injury was $\mathrm{C} 5$, similar to findings in Italy. ${ }^{4}$ The associated most common neurological level of $\mathrm{C} 4$ again confirmed the severity of these injuries, with the most common neurological level from the Italian study being C6, indicating more severe injuries encountered here. The level was determined using the ASIA scoring system with the neurological level being the last one with normal ( 5 out of 5) power compared with the Frankel grading system ${ }^{7}$ using the last level as the one with 3 out of 5 power. The average stay of 30 days compares well with the Italian diving accident study ${ }^{4}$ in which the average stay was 60 days, indicating a quick and effective turnover of patients.

Several factors were identified that contribute to diving injuries. Not knowing, or the inability to judge, the water depth plays a very important role. An Australian study showed that the minimum depth required for a trained diver taking a running dive is 1.22 metres; $89 \%$ of injuries occurred in water $<1.52 \mathrm{~m}$ deep, but rarely in water of 0.46 $-0.61 \mathrm{~m}$, indicating that common sense prevails in most cases. ${ }^{6}$ They recommend safety practices such as steering up to the surface when diving, head protection with extended arms and diving only when necessary. Only $54 \%$ of patients in this study used outstretched hands during the dive, making diving technique another important factor to be addressed in a prevention campaign. The sea, tidal pools and even rivers are subject to tidal change where, within 6 hours, an almost $2 \mathrm{~m}$ 


\section{Original ArTiCles}

depth change can occur, which is a common causative factor in diving injuries. Further contributing factors include diving into turbid water, diving into a wave and alcohol abuse.

\section{Conclusion}

Our study provides a very specific target group and locations to address in a prevention programme. An effective programme should target young males intensely during the summer months and focus specifically on alcohol abuse and diving techniques. Education in schools could address the target group and provide exposure to large groups of people; and by using volunteer victims of diving injuries, the impact may be even greater. Further emphasis must be placed on local area education, with warning signs and lifeguards identifying safe areas in which to swim. A quick transfer to a specialised spinal injury unit would decrease associated morbidities such as bedsores and pneumonia.

The current diving injury awareness campaign only includes an advertisement with high rotation during the summer months; this should be broadened to an extensive prevention programme including radio advertisements and structured school education programmes, and should target group-specific injury prevention.

\section{References}

1. American Spinal Injury Association/International Medical Society of Paraplegia. International Standards for Neurological and Functional Classification of Spinal Cord Injury. Revised 1996. Chicago: American Spinal Injury Association, 1996.

2. De Vivo MJ, Sekar P. Prevention of spinal cord injuries that occur in swimming pools. Spinal Cord 1997;35(8):509-515.

3. Korres DS, Benetos IS, Themistocleous GS, Avrogenis AF, Nikolakakos L, Liantis PT. Diving injuries of the cervical spine in amateur divers. Spine J 2006;6(1): 44-49 (Epub 6 December 2005).

4. Aito S, D’Andrea M, Werhagen L. Spinal cord injuries due to diving accidents. Spinal Cord 2005;43(2):109-116.

5. Barss P, Djerrari H, Leduc BE, Lepage Y, Dionne CE. Risk factors and prevention for spinal cord injury from diving in swimming pools and natural sites in Quebec, Canada: a 44-year study. Accid Anal Prev 2008;40(2):787-797 (Epub 8 October 2007).

6. Blanksby BA, Wearne FK, Elliott BC, Blitvich JD. Aetiology and occurrence of diving injuries. A review of diving safety. Sports Med 1997;23(4):228-246.

7. Frankel HL, Hancock DO, Hyslop G, et al. The value of postural reduction in the initial management of closed injuries of the spine with paraplegia and tetraplegia. Paraplegia 1969;7:179-192. 